\title{
Immunohistochemical Study of NR2C2, BTG2, TBX19, and CDK2 Expression in 31 Paired Primary/Recurrent Nonfunctioning Pituitary Adenomas
}

\author{
Xiaohui Yao, ${ }^{1}$ Yazhuo Zhang $\mathbb{D},{ }^{2}$ Lijuan $W u,{ }^{1}$ Rui Cheng, \\ Chuzhong Li, ${ }^{2}$ Chongxiao Qu, ${ }^{1}$ and Hongming Ji ${ }^{1}{ }^{1}$ \\ ${ }^{1}$ Shanxi Provincial People's Hospital, Taiyuan, Shanxi Province, China \\ ${ }^{2}$ Key Laboratory of Central Nervous System Injury Research, Beijing Neurosurgical Institute, Capital Medical University, Beijing, China
}

Correspondence should be addressed to Hongming Ji; jihongmingjhm@yeah.net

Received 6 October 2018; Revised 12 March 2019; Accepted 3 April 2019; Published 16 May 2019

Academic Editor: Giuseppe Reimondo

Copyright (c) 2019 Xiaohui Yao et al. This is an open access article distributed under the Creative Commons Attribution License, which permits unrestricted use, distribution, and reproduction in any medium, provided the original work is properly cited.

This study investigated potential markers for predicting nonfunctioning pituitary adenoma (NFPA) invasion and recurrence by high-throughput tissue microarray analyses. We retrospectively studied two groups of patients: 60 nonrecurrent NFPA cases that included noninvasion and invasion subtypes and 43 recurrent cases that included primary NFPA. A total of 31 paired patient samples were evaluated (12 patients with one surgery and 31 who had undergone two operations, with both tumors analyzed). Expressions of nuclear receptor subfamily 2 group C member 2 (NR2C2), B cell translocation gene 2, T-box-19 (TBX19), and cyclin-dependent kinase 2 (CDK2) in surgically resected specimens were assessed by immunohistochemistry. The relationships between marker expression and clinical characteristics including age, sex, tumor volume, and follow-up time were analyzed. Tumor volume and invasion as well as follow-up time were significantly associated with invasion and recurrence $(\mathrm{P}<0.01)$. Of the 60 nonrecurrent samples, $15 / 41$ and 13/19 showed high NR2C2 expression in the noninvasion and invasion groups, respectively $\left(\chi^{2}=5.287, \mathrm{P}=\right.$ 0.021). NR2C2 was also overexpressed in 43 primary recurrent cases $\left(\chi^{2}=5.433, \mathrm{P}=0.02\right)$, whereas CDK2 $\left(\chi^{2}=11.242, \mathrm{P}=0.001\right)$ and TBX19 $\left(\chi^{2}=4.875, \mathrm{P}=0.027\right)$ were downregulated. In the 31 paired samples, NR2C2 was more highly expressed in the recurrent as compared to the primary tumor. High NR2C2 expression was associated with NFPA invasion, recurrence, and progression, while TBX19 and CDK2 were associated with NFPA recurrence.

\section{Introduction}

Pituitary adenomas are common and benign intracranial neoplasms, of which $14 \%-28 \%$ are nonfunctioning pituitary adenomas (NFPAs) [1]. NFPA are the most common type of adenomas when taking into account only macroadenomas. Although they typically grow slowly, some have an aggressive character and invade adjacent dura mater and surrounding bones before reaching the cavernous sinuses. This makes complete resection difficult, leading to postoperative recurrence or regrowth. Even after complete or near-complete surgical resection, $12 \%-58 \%$ of NFPA patients experience regrowth within 5 years, which cannot be effectively controlled by available therapeutics [2-5]. In contrast to functioning adenomas for which several effective and relatively safe targeted pharmacological therapies have been developed, a specific medical treatment for NFPA is still lacking [6]. The new WHO classification underscores the adoption of a pituitary adenohypophyseal cell lineage as the main principle guiding the classification of adenomas. The main technique for tumor classification is immunohistochemistry with the combination of immunostains for the main pituitary hormones and, when required, pituitary transcription factors [7]. Therefore, in order to develop improved treatment strategies, appropriate biomarkers for NFPA behavior must be identified. Various markers for the aggressive behavior of NFPA have been reported, including altered expression of nuclear receptor superfamily 2 group C member 2 (NR2C2), B cell translocation gene 2 (BTG2), TBX19, and cyclindependent kinase 2 (CDK2), but the relationship between 
histopathological features of the disease and clinical outcome is unclear [8-11].

NR2C2 is extensively expressed in the ovary, testis, and brain [12] and regulates different aspects of neuronal development [13]; it also induces tumor initiation under the control of various modulators [14, 15]. BTG2 is a member of the BTG/TOB protein family and is encoded by an early growth response gene. Abnormal BTG2 expression has been implicated in tumor development and progression. T-box (TBX19) (also referred to as TPIT) is a transcription factor that is expressed in the pituitary gland; the TBX protein family plays important roles in early embryogenesis [16] and is associated with the development of various malignancies [17]. CDK2 is an enzyme that mediates the G1/S transition, and its dysregulation contributes to tumorigenesis. Human CDK2 is expressed in the pituitary pars intermedia, likely through a mechanism that is active in cells with elevated proliferative activity [18].

At present, predicting NFPA behavior such as progression and recurrence remains challenging. To this end, we investigated the correlation between NR2C2, BTG, TBX19, and CDK2 and NFPA invasiveness and recurrence by immunohistochemical and tissue microarray (TMA) analyses.

\section{Materials and Methods}

2.1. Patients. We retrospectively reviewed 134 NFPA cases including 60 nonrecurrent patients at Shanxi Province People's Hospital and 43 primary recurrent NFPA cases at the Beijing Tiantan Hospital of Capital Medical University; among them, paired samples were available for 31 patients (12 with one surgery and 31 who had undergone two operations, with both tumors analyzed). The patients were all diagnosed with NFPA according to the 2007 World Health Organization histological classification from January 2008 to December 2014 and underwent transsphenoidal or transcranial operation that was followed up with magnetic resonance imaging. Demographic information such as sex and age; clinical information including tumor invasion, size, and volume; and clinical symptoms such as headaches, diminution of visual acuity, and defective field of vision were recorded for each patient.

The patients were classified into two groups. The nonrecurrent group included noninvasive (Knosp classification I and II) and invasive (Knosp classification IIIb and IV) tumors [19]. The recurrent group included 43 primary recurrent tumors, of which 31 were paired (primary and recurrent tumors). The quality of 12 specimens from the second operation was too poor for TMA, so only 31 paired primary and recurrent tumor specimens were included in the analysis. Recurrence was defined as a new lesion after complete remission or enlargement of a residual tumor, as confirmed histologically by a neurosurgeon and two neuroradiologists who were blinded to patient information. It has been documented that $10-20 \%$ of completely resected tumors recur after 5-10 years and when residual tumor remains after surgery, the percentage can be up to $50 \%$ within 5-10 years [20-22]. Although it is difficult to define nonrecurrence, in our study, the patients who were followed up for more than 6 years where there was no recurrence were defined as nonrecurrence. Compared to recurrence, it hints that the tumor did not recur temporarily and it also indicates that the tumor progresses slowly. This study was approved by the institutional review board and patients provided written, informed consent for their participation.

2.2. TMA Construction. TMAs were prepared using the BOND-III fully automated array instrument (Leica Biosystems, Wetzlar, Germany) from three $2.0 \mathrm{~mm}$ in diameter core biopsies of a representative tumor which were randomly ordered on the array. The TMA was cut into $4 \mu \mathrm{m}$ sections on a microtome, placed in a water bath at $50^{\circ} \mathrm{C}$, and then transferred to positively charged glass slides. The specimens were deparaffinized and rehydrated through a graded series of alcohol with water, dried at room temperature for $24-48 \mathrm{~h}$, and stored at $-80^{\circ} \mathrm{C}$ until use.

2.3. Immunohistochemistry. The content and quality of samples on the TMA slides were confirmed by hematoxylin and eosin staining. TMAs were processed for immunohistochemistry with the automatic array device. Other carcinoma and pituitary tissue specimens were used as positive and negative controls, respectively. NFPA samples were mostly gonadotrophic tumors but also included silent tumors of other lineages and occasionally null cell tumors. Therefore, we included antibodies against growth hormone, prolactin, thyroid-stimulating hormone, adrenocorticotropic hormone (ACTH), follicle-stimulating hormone, and luteinizing hormone in the immunohistochemical analysis along with antibodies against the following proteins: NR2C2 (1:600), with $15 \mathrm{~min}$ each of alkaline epitope retrieval (ER) and heat-induced epitope retrieval (HIRE); BTG2 (1:50), with $30 \mathrm{~min}$ of acid ER and $15 \mathrm{~min}$ of HIRE; TBX19 (8:1), with $30 \mathrm{~min}$ of alkaline ER and $15 \mathrm{~min}$ of HIRE; and CDK2 (1:600), with $20 \mathrm{~min}$ of alkaline ER and $15 \mathrm{~min}$ of HIRE. The primary antibodies were from Abcam (Cambridge, MA, USA) and were detected with the Bond Polymer Refine Detection kit (DS9800; Leica Biosystems, Wetzlar, Germany). Digital images were acquired using an Aperio AT2 scanner (Leica Biosystems). Slides were independently evaluated and scored by two neuropathologists who were blinded to group assignment. Differences in interpretation were resolved by consensus.

2.4. TMA Scanning and Image Analysis. Immunopositive cells in 1000 tumors were counted under a light microscope by two neuropathologists; the positively stained area was expressed relative to the whole adenoma area. Staining intensity was scored as 0 (negative), 1 (weak), 2 (moderate), or 3 (strong). Immunopositivity for each biomarker was scored according to the following semiquantitative scale: 0 (no reactivity), 1 (1\%-25\% of neoplastic cells are positive), 2 (26\%-50\% positive), 3 (51\%-75\% positive), and 4 (76\%-100\% positive). The 12 scores were summed for statistical analyses. Tumors with a final staining score $\geq 6$ were considered as having high expression, whereas a score $<6$ was considered as low expression. 
TABLE 1: Characteristics of the cohort.

\begin{tabular}{|c|c|c|c|c|c|c|c|}
\hline & $\begin{array}{c}\text { Noninvasion } \\
\mathrm{N}=41\end{array}$ & $\begin{array}{c}\text { Invasion } \\
\mathrm{N}=19\end{array}$ & $\mathrm{P}$ & $\begin{array}{c}\text { Nonrecurrence } \\
\qquad \mathrm{N}=60\end{array}$ & $\begin{array}{l}\text { Recurrence } \\
\mathrm{N}=43\end{array}$ & $\mathrm{P}$ & $\begin{array}{l}\text { Paired- } \\
\text { recurrence } \\
\mathrm{N}=31\end{array}$ \\
\hline Age & $52.00 \pm 10.20$ & $54.05 \pm 11.34$ & 0.488 & $52.65 \pm 10.54$ & $40.84 \pm 12.93$ & $<0.001$ & $37.48 \pm 11.58$ \\
\hline $\operatorname{Sex}(M / F)$ & $25 / 16$ & $6 / 13$ & 0.034 & $31 / 29$ & $14 / 29$ & 0.054 & $11 / 20$ \\
\hline Volume & $\begin{array}{c}3591 \\
(1680-6292)\end{array}$ & $\begin{array}{c}8120 \\
(5288-12818)\end{array}$ & $<0.001$ & $\begin{array}{c}4885 \\
(2078-8085)\end{array}$ & $\begin{array}{c}7936 \\
(5240-14548)\end{array}$ & 0.001 & $\begin{array}{c}10140 \\
(6125-14548)\end{array}$ \\
\hline $\begin{array}{l}\text { Maximum } \\
\text { diameter }\end{array}$ & $22.83 \pm 5.73$ & $32.00 \pm 9.64$ & 0.001 & $25.73 \pm 8.31$ & $31.05 \pm 9.69$ & 0.004 & $31.94 \pm 9.73$ \\
\hline Invasion (N/Y) & - & - & - & $41 / 19$ & $17 / 26$ & 0.004 & $12 / 19$ \\
\hline Follow-up time & $44.41 \pm 17.93$ & $37.26 \pm 16.98$ & 0.150 & $42.15 \pm 17.81$ & $31.81 \pm 16.04$ & 0.003 & $31.65 \pm 15.80$ \\
\hline
\end{tabular}

TABLE 2: Noninvasion group versus invasion group and nonrecurrent group versus primary recurrent group.

\begin{tabular}{|c|c|c|c|c|c|c|c|c|c|}
\hline & & Noninvasion & Invasion & $\mathrm{X}^{2}$ & $\mathrm{P}$ & Nonrecurrence & Primary recurrence & $\mathrm{X}^{2}$ & $\mathrm{P}$ \\
\hline \multirow[t]{2}{*}{ NR2C2 } & Low- & $26(63.4 \%)$ & $6(31.6 \%)$ & 5.287 & 0.021 & $32(53.3 \%)$ & $13(30.2 \%)$ & 5.433 & 0.020 \\
\hline & High- & $15(36.6 \%)$ & $13(68.4 \%)$ & & & $28(46.7 \%)$ & $30(69.8 \%)$ & & \\
\hline \multirow[t]{2}{*}{ BTG2 } & Low- & $35(85.4 \%)$ & $18(94.7 \%)$ & 0.384 & 0.536 & $53(88.3 \%)$ & $43(100 \%)$ & 3.698 & 0.054 \\
\hline & High- & $6(14.6 \%)$ & $1(5.3 \%)$ & & & $7(11.7 \%)$ & $0(0 \%)$ & & \\
\hline \multirow[t]{2}{*}{$\mathrm{CDK} 2$} & Low- & $1(2.4 \%)$ & $1(5.3 \%)$ & - & 0.537 & $2(3.3 \%)$ & $11(25.6 \%)$ & 11.242 & 0.001 \\
\hline & High- & $40(97.6 \%)$ & $18(94.7 \%)$ & & & $58(96.7 \%)$ & $32(74.4 \%)$ & & \\
\hline \multirow[t]{2}{*}{ TBX19 } & Low- & $31(75.6 \%)$ & $11(57.9 \%)$ & 1.940 & 0.164 & $42(70.0 \%)$ & $38(88.4 \%)$ & 4.875 & 0.027 \\
\hline & High- & $10(24.4 \%)$ & $8(42.1 \%)$ & & & $18(30.0 \%)$ & $5(11.6 \%)$ & & \\
\hline
\end{tabular}

2.5. Statistical Analysis. Statistical analysis was performed using SPSS v.20.0 software (IBM Inc., Armonk, NY, USA). Results for categorical variables are presented as proportions and frequencies. Differences in categorical variables among groups were analyzed with the $\chi^{2}$ test or Fisher's exact test. Descriptive statistics are presented as the mean \pm standard deviation. Comparisons between groups were made with the unpaired two-sample $t$-test. Tumor volume is presented as a median value with a range. Comparisons between groups were made with the Mann-Whitney test. Statistical significance was defined as $\mathrm{P}<0.05$.

\section{Results}

3.1. Characteristics of the Study Cohort. Of the 134 NFPA cases included in the study, 60 were diagnosed as nonrecurrent and 43 were primary recurrent, and paired samples were available for 31 patients. An endoscopic endonasal transsphenoidal approach was used in 87 cases (64.9\%), a microscopic transsphenoidal approach in 37 cases $(27.6 \%)$, and a craniotomy in 10 cases (7.5\%). Mean patient age was 47 years (range, 31-59.5 years), and there were 45 men and 58 women. Chief complaints at the time of presentation were visual disturbance $(n=66 ; 64.1 \%)$, headache $(n=54 ; 52.43 \%)$, and visual field deficits $(n=20 ; 19.4 \%)$. Up to the end of the follow-up period (December 31, 2014), 43 patients had experienced recurrence after surgery; the mean duration $( \pm$ SD) of follow-up was $31.81 \pm 16.04$ months.

We evaluated the interdependence of clinical parameters in nonrecurrent and recurrent primary patients with the unpaired two-sample $t$-test, Mann-Whitney test, and $\chi^{2}$ test. Parameters related to prognosis were selected based on our clinical experience. No patients underwent adjuvant radiotherapy during follow-up. We found that tumor volume and maximum diameter were associated with invasion and recurrence $(\mathrm{P}<0.01)$, whereas invasion, tumor volume and maximum diameter, and follow-up time were associated with recurrence $(\mathrm{P}<0.01$; Table 1$)$. This indicated that large tumors are more likely to be invasive and to recur and that invasive tumors have a greater risk of recurrence. There were no differences with respect to sex or age among groups.

3.2. NR2C2, BTG2, CDK2, and TBX19 Expression and Correlation with Adenoma. Of the 60 nonrecurrent samples, 15/41 and 13/19 showed high NR2C2 expression in the noninvasive and invasive NFPA groups, respectively $\left(\chi^{2}=5.287, \mathrm{P}=0.021\right.$, Figure 1). CDK2 was upregulated, whereas BTG and TBX19 were downregulated in noninvasive and invasive groups, respectively. However, there were no differences in the levels of the three proteins between these two groups (Table 2).

Of the 43 cases of recurrent primary NFPA, 30 overexpressed NR2C2 as compared to 28/60 in the nonrecurrent group. NR2C2 was highly expressed in the primary recurrent group ( $\chi^{2}=5.433, \mathrm{P}=0.02$, Figure 1$)$. On the other hand, lowly expressed CDK2 was observed in $2 / 60$ patients in the nonrecurrence group and in $11 / 32$ patients in the primary recurrent group ( $\chi^{2}=11.242, \mathrm{P}=0.001$, Figure 2$)$. TBX19 was also expressed at a low level in 38/43 recurrent primary tumors $\left(\chi^{2}=4.875, \mathrm{P}=0.027\right.$, Figure 2$)$. BTG was also downregulated in nonrecurrent and primary recurrent NFPA, but there was no statistically significant difference between the 


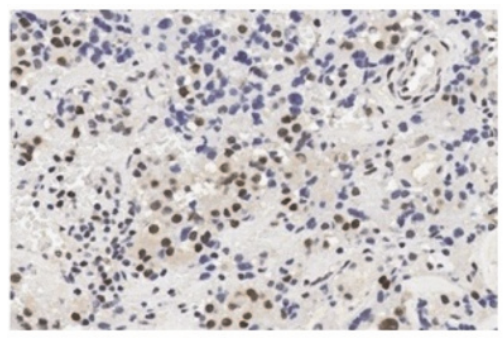

(Aa)

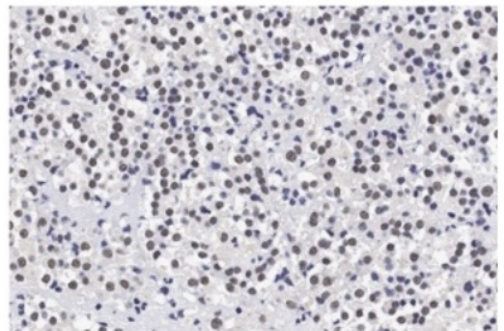

(Ba)

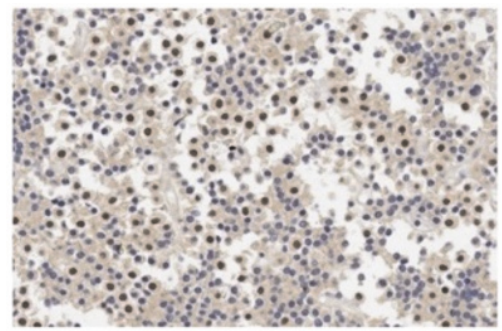

(Ca)

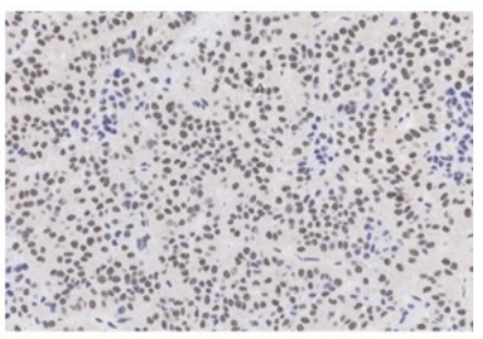

$(\mathrm{Ab})$

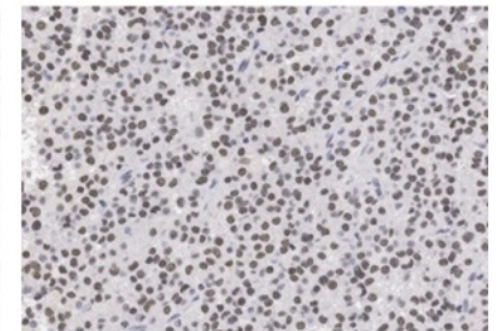

$(\mathrm{Bb})$

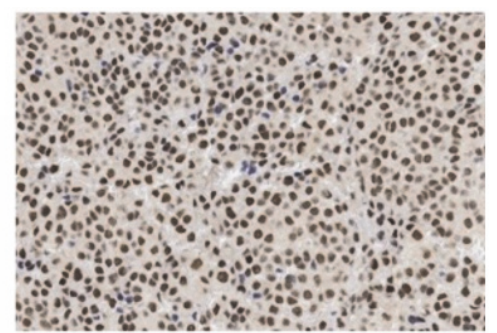

$(\mathrm{Cb})$

FIGURE 1: NR2C2 expressed in each group. (Aa): lowly expressed in noninvasive group; $(\mathrm{Ab})$ : highly expressed in invasive group; (Ba): lowly expressed in nonrecurrent group; $(\mathrm{Bb})$ : highly expressed in primary recurrent group; (Ca): lowly expressed in primary group; (Cb): highly expressed in recurrent group.

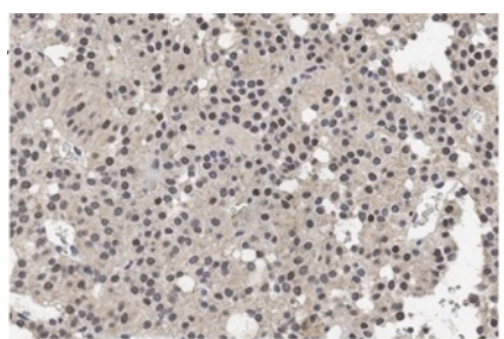

(Aa)

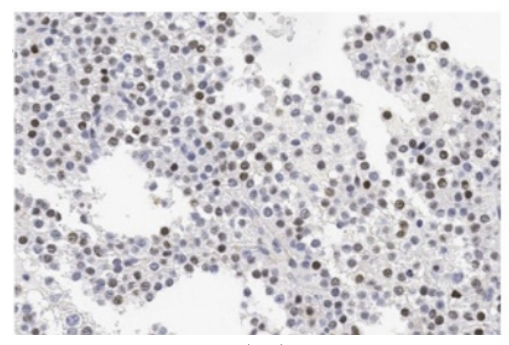

(Ba)

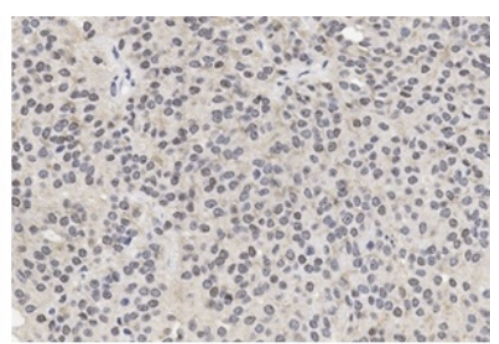

$(\mathrm{Ab})$

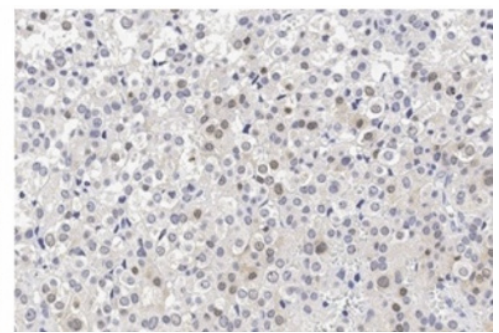

$(\mathrm{Bb})$

FIGURE 2: BTG2 and CDK2 expressed in each group. (Aa): BTG2 highly expressed in primary recurrent group; (Ab): BTG2 lowly expressed in nonrecurrent group. (Ba): CDK2 highly expressed in primary recurrent group; (Bb): CDK2 lowly expressed in nonrecurrent group. 
TABLE 3: The paired recurrent group.

\begin{tabular}{|c|c|c|c|c|c|}
\hline \multicolumn{6}{|c|}{ Recurrence } \\
\hline Primary & & Low- & High- & $\mathrm{X}^{2}$ & $\mathrm{P}$ \\
\hline \multirow[t]{2}{*}{ NR2C2 } & Low- & $1(14.3 \%)$ & $6(85.7 \%)$ & 4.167 & 0.031 \\
\hline & High- & $0(0 \%)$ & $24(100 \%)$ & & \\
\hline \multirow[t]{2}{*}{ BTG2 } & Low- & 31 & 0 & - & - \\
\hline & High- & 0 & 0 & & \\
\hline \multirow[t]{2}{*}{ CDK2 } & Low- & $7(77.8 \%)$ & $2(22.2 \%)$ & - & 1.000 \\
\hline & High- & $1(4.5 \%)$ & $21(95.5 \%)$ & & \\
\hline \multirow[t]{2}{*}{ TBX19 } & Low- & $20(71.4 \%)$ & $8(28.6 \%)$ & 2.500 & 0.109 \\
\hline & High- & $2(66.7 \%)$ & $1(33.3 \%)$ & & \\
\hline
\end{tabular}

two groups. Thus, primary recurrent tumors showed low expression of CDK2 and TBX19 but overexpression of NR2C2 (Table 2).

In 31 paired specimens, recurrent tumors showed higher NR2C2 expression than primary tumors (30/31 versus $24 / 31$, $\chi^{2}=4.167, \mathrm{P}=0.031$; Figure 1$)$. There were no differences in BTG, CDK2, and TBX19 expression between the two groups, although BTG and TBX19 were downregulated and CDK2 was upregulated. These results indicated that NR2C2 overexpression can be used to distinguish recurrent from primary NFPA tumors (Table 3 ).

\section{Discussion}

Surgery is the primary treatment in NFPAs. However, at least half of the cases result in subtotal or partial resection leading to significant rates of recurrence. Many unanswered questions remain regarding the management of recurrent NFPAs including surgical approach and medicine therapy [23]. Owing to its invasiveness and progression, pituitary adenoma-especially NFPA-is difficult to resect and treat. Recently, the term "pituitary neuroendocrine tumor" was proposed for pituitary adenoma to emphasize its unpredictable nature [24]. However, the relationship between molecular alterations and clinical outcome in pituitary disorders is not well understood. To address this issue, we examined the association between the expression of four biomarkers and tumor invasion and recurrence along with other clinical characteristics such as tumor diameter and volume and follow-up time. The results of our study showed that visual disturbances due to compression of the optic apparatus were common, as were headache and visual field deficits. Moreover, large tumors were more likely to be invasive and to recur, while the presence of invasive tumors and a longer follow-up time increased the risk of recurrence.

NR2C2, also known as testicular nuclear receptor 4, was first cloned from human hypothalamus, prostate, and testes [25]. NR2C2 plays an important role in the central nervous system, regulating many physiological and pathophysiological processes [26]. Phosphorylation and/or dephosphorylation, acetylation, cyclic AMP/protein kinase A signaling, a vitamin A metabolite, forkhead box O3a, and other factors were shown to suppress or enhance NR2C2 function in diverse biological processes [14]. NR2C2 can both inhibit and activate target genes depending on the physiological context [27]. For example, it was shown to stimulate the migration of liver cancer cells but suppressed liver cancer cell growth [28]. In breast cancer, NR2C2 interacts with estrogen receptor to inhibit MCF-7 cell proliferation [29]. NR2C2 is highly expressed in CD133+ prostate cancer stem/progenitor cells and promoted their invasion through enhancer of zeste homolog 2 signaling involving several metastasis-associated genes such as NOTCH1, SLUG, transforming growth factor $\beta 1$, and matrix metalloproteinase 9 [30]. NR2C2 was overexpressed in pituitary adenoma and promoted ACTH secretion, cell proliferation, and tumor invasion [31]. In this study, we observed a clear association between elevated NR2C2 expression and tumor invasion and recurrence. Thus, NR2C2 plays an important role in NFPA invasion, development, and progression.

BTG2 functions as a tumor suppressor or promoter in various human malignancies [32]. Low BTG2 expression was linked to high tumor grade, size, and recurrence in estrogen receptor-positive breast cancer [33]. Additionally, BTG2 was downregulated in renal cell carcinoma as compared to normal control renal tissue, whereas BTG2 overexpression was associated with inhibition of cell growth, migration, and invasion [34]. A decrease in BTG2 level could indirectly activate $\mathrm{AKT}$ and extracellular signal-regulated kinase/mitogenactivated protein kinase signaling pathways and regulate the downstream effects of miR-25-3p to stimulate proliferation of triple-negative breast cancer cells [35]. Our results demonstrated that BTG2 expression was reduced in invasive, primary, and recurrent NFPA, suggesting a tumor-suppressor function.

TBX19 is involved in early embryogenesis, cell differentiation, and organogenesis of the pituitary gland and has also been implicated in carcinogenesis. TBX transcription factors are transcriptional activators or repressors [36]. TBX19 was shown to be more highly expressed in colorectal cancer tissue, which was associated with lymph node metastasis [37]. Meanwhile, it was also reported that TBX19 acts downstream of KRAS in human cancer [38]. TBX19 was the first TBX family member to be identified in the pituitary; it was shown to repress the gonadotrophic phenotype possibly through gonadotrophic-specific splicing factor $1[36,39]$ 
and thus modulate terminal differentiation of pituitary lineages. Specifically, TBX19 regulated the proopiomelanocortin lineage that gives rise to corticotropes. In the intermediate pituitary, TBX19 is essential for intermediate lobe and melanotrope differentiation and is expressed in paired-box 7-positive cells that are associated with suppression of (sex determining region Y)-box 2 and induction of differentiation. Thus, TBX19 is required for the terminal differentiation of pituitary proopiomelanocortin-expressing cells [40, 41]. In this study, TBX19 level was decreased in the primary recurrent group, suggesting a role in the recurrence of NFPA. However, given that it was also expressed at a low level in the invasion and recurrence groups, it could also act as a transcriptional repressor in the development of NFPA.

CDK2 regulates cell cycle progression and its activity depends on the binding of regulatory cyclin subunits. Inappropriate expression of CDK2 has been implicated in various malignancies including lung carcinoma, pancreatic carcinoma, ovarian carcinoma, and sarcomas [42]. CDK2 promoted lineage commitment of neuron-glial antigen 2expressing progenitors in the adult subventricular zone and induced the differentiation of adult neural progenitor cells and oligodendroglia [43]. However, other studies have shown that CDK2 is not essential for neural progenitor cell proliferation and differentiation and hippocampal granule neuron survival in vivo [44], implying that CDK2 activity is contextspecific. Low-to-moderate CDK2 expression is related to the risk of colon cancer, although CDK2 activity above a certain threshold may also pose a risk. Some cancers may transition through a low-CDK2 state at early, possibly benign periods of growth. In support of this "two-state" hypothesis, it was reported that only complete ablation of CDK2 blocked tumorigenesis [45]. We found here that CDK2 was downregulated in the primary NFPA group, suggesting that this is a marker for NFPA development; on the other hand, CDK2 expression was elevated in the invasion and recurrence groups, implying that a high CDK2 level contributes to tumor progression. This dichotomous role makes NFPA an attractive therapeutic target for NFPA treatment.

\section{Conclusions}

In this study, we found that NR2C2 expression is associated with the invasion, recurrence, and progression of NFPA. TBX19 and CDK2 were also found to be involved in NFPA recurrence. This suggests that therapeutic strategies targeting NR2C2 can suppress NFPA development, whereas those targeting TBX19 and CDK2 may prevent its regrowth. However, whether these three factors interact to promote NFPA and the detailed mechanism of this interaction remains to be elucidated. More work is needed to determine whether these factors interact to promote other types of pituitary adenoma.

\section{Data Availability}

The analyzed datasets generated during the study are available from the corresponding author upon reasonable request.

\section{Ethical Approval}

This study was approved by the ethics committee of our hospital and did not cause extra harm and additional medical cost and is strictly in accordance with the principles of ethics.

\section{Consent}

All patients have read an informed consent form and had the opportunity to ask questions and all questions have been answered. The participation in this study is voluntary and all patients signed the informed consent.

\section{Conflicts of Interest}

The authors declare that there are no conflicts of interest.

\section{Authors' Contributions}

Xiaohui Yao designed the study and conducted the experiments and drafted the manuscript. Yazhuo Zhang and Hongming Ji designed the study and conducted the experiments. Lijuan $\mathrm{Wu}$ contributed to the acquisition and collection of the data. Chuzhong $\mathrm{Li}$ and Chongxiao $\mathrm{Qu}$ were responsible for analyzing and interpreting the data and drafted the manuscript. All authors read and approved the final manuscript.

\section{Acknowledgments}

This work was sponsored by the National High Technology Research and Development Program of China (863 Program) (2014AA020610) and the Research Special Fund for Public Welfare Industry of Health (201402008).

\section{References}

[1] A. F. Daly, M. Rixhon, C. Adam, A. Dempegioti, M. A. Tichomirowa, and A. Beckers, "High prevalence of pituitary adenomas: a cross-sectional study in the province of Liège, Belgium," The Journal of Clinical Endocrinology \& Metabolism, vol. 91, no. 12, pp. 4769-4775, 2006.

[2] E. P. O'Sullivan, C. Woods, N. Glynn et al., “The natural history of surgically treated but radiotherapy-naïve nonfunctioning pituitary adenomas," Clinical Endocrinology, vol. 71, no. 5, pp. 709-714, 2009.

[3] A. Colao, C. di Somma, R. Pivonello, A. Faggiano, G. Lombardi, and S. Savastano, "Medical therapy for clinically nonfunctioning pituitary adenomas," Endocrine-Related Cancer, vol. 15, no. 4, pp. 905-915, 2008.

[4] E. Ferrante, M. Ferraroni, T. Castrignanò et al., "Nonfunctioning pituitary adenoma database: a useful resource to improve the clinical management of pituitary tumors," European Journal of Endocrinology, vol. 155, no. 6, pp. 823-829, 2006.

[5] O. M. Dekkers, A. M. Pereira, F. Roelfsema et al., "Observation alone after transsphenoidal surgery for nonfunctioning pituitary macroadenoma," The Journal of Clinical Endocrinology \& Metabolism, vol. 91, no. 5, pp. 1796-1801, 2006.

[6] M. Mercado, V. Melgar, L. Salame, and D. Cuenca, "Clinically non-functioning pituitary adenomas: Pathogenic, diagnostic 
and therapeutic aspects," Endocrinologia, Diabetes y Nutricion, vol. 64, no. 7, pp. 384-395, 2017.

[7] M. B. S. Lopes, "The 2017 World Health Organization classification of tumors of the pituitary gland: a summary," Acta Neuropathologica, vol. 134, no. 4, pp. 521-535, 2017.

[8] F. Salehi, A. Agur, B. W. Scheithauer, K. Kovacs, R. V. Lloyd, and M. Cusimano, "Biomarkers of pituitary neoplasms: A review (Part II)," Neurosurgery, vol. 67, no. 6, pp. 1790-1798, 2010.

[9] G. Zada, W. W. Woodmansee, S. Ramkissoon, J. Amadio, V. Nose, and E. R. Laws Jr., "Atypical pituitary adenomas: incidence, clinical characteristics, and implications," Journal of Neurosurgery, vol. 114, no. 2, pp. 336-344, 2011.

[10] H. Nishioka, N. Inoshita, O. Mete et al., "The complementary role of transcription factors in the accurate diagnosis of clinically nonfunctioning pituitary adenomas," Endocrine Pathology, vol. 26, no. 4, pp. 349-355, 2015.

[11] C. Tanase, R. Albulescu, E. Codrici et al., "Decreased expression of APAF-1 and increased expression of cathepsin B in invasive pituitary adenoma," OncoTargets and Therapy, vol. 8, pp. 81-90, 2014.

[12] A. L. Bookout, Y. Jeong, M. Downes, R. T. Yu, R. M. Evans, and D. J. Mangelsdorf, "Anatomical profiling of nuclear receptor expression reveals a hierarchical transcriptional network," Cell, vol. 126, no. 4, pp. 789-799, 2006.

[13] Y.-T. Chen, L. L. Collins, H. Uno et al., "Abnormal cerebellar cytoarchitecture and impaired inhibitory signaling in adult mice lacking TR4 orphan nuclear receptor," Brain Research, vol. 1168, no. 1, pp. 72-82, 2007.

[14] S.-J. Lin, S. O. Lee, Y.-F. Lee et al., “TR4 nuclear receptor functions as a tumor suppressor for prostate tumorigenesis via modulation of DNA damage/repair system," Carcinogenesis, vol. 35, no. 6, pp. 1399-1406, 2014.

[15] S. Lin, D. Yang, G. Yang et al., "TR2 and TR4 orphan nuclear receptors," in Nuclear Receptors in Development and Disease, vol. 125 of Current Topics in Developmental Biology, pp. 357-373, Elsevier, 2017.

[16] V. E. Papaioannou, "The t-box gene family: Emerging roles in development, Stem cells and cancer," Development, vol. 141, no. 20, pp. 3819-3833, 2014.

[17] S. Wansleben, J. Peres, S. Hare, C. R. Goding, and S. Prince, "T-box transcription factors in cancer biology," Biochimica et Biophysica Acta (BBA) - Reviews on Cancer, vol. 1846, no. 2, pp. 380-391, 2014.

[18] E. Macias, P. L. Miliani De Marval, A. Senderowicz, J. Cullen, and M. L. Rodriguez-Puebla, "Expression of CDK4 or CDK2 in mouse oral cavity is retained in adult pituitary with distinct effects on tumorigenesis," Cancer Research, vol. 68, no. 1, pp. 162-171, 2008.

[19] A. S. G. Micko, A. Wöhrer, S. Wolfsberger, and E. Knosp, "Invasion of the cavernous sinus space in pituitary adenomas: Endoscopic verification and its correlation with an MRI-based classification," Journal of Neurosurgery, vol. 122, no. 4, pp. 803811, 2015.

[20] Y. Chen, C. D. Wang, Z. P. Su et al., "Natural history of postoperative nonfunctioning pituitary adenomas: A systematic review and meta-analysis," Neuroendocrinology, vol. 96, no. 4, pp. 333342, 2012.

[21] S. Brochier, F. Galland, M. Kujas et al., "Factors predicting relapse of nonfunctioning pituitary macroadenomas after neurosurgery: a study of 142 patients," European Journal of Endocrinology, vol. 163, no. 2, pp. 193-200, 2010.
[22] Z. H. A. Sadik, E. H. J. Voormolen, P. R. A. M. Depauw et al., "Treatment of nonfunctional pituitary adenoma postoperative remnants: adjuvant or delayed gamma knife radiosurgery?" World Neurosurgery, vol. 100, pp. 361-368, 2017.

[23] P. D. Delgado-López, J. Pi-Barrio, M. T. Dueñas-Polo, M. Pascual-Llorente, and M. C. Gordón-Bolaños, "Recurrent nonfunctioning pituitary adenomas: a review on the new pathological classification, management guidelines and treatment options," Clinical and Translational Oncology, vol. 20, no. 10, pp. 1233-1245, 2018.

[24] S. L. Asa and O. Mete, "What's new in pituitary pathology?" Histopathology, vol. 72, no. 1, pp. 133-141, 2018.

[25] C. Chang, S. L. Da Silva, R. Ideta, Y. Lee, S. Yeh, and J. P. H. Burbach, "Human and rat TR4 orphan receptors specify a subclass of the steroid receptor superfamily," Proceedings of the National Acadamy of Sciences of the United States of America, vol. 91, no. 13, pp. 6040-6044, 1994.

[26] S. E. Mullican, J. R. DiSpirito, and M. A. Lazar, "The orphan nuclear receptors at their 25-year reunion," Molecular Endocrinology, vol. 51, no. 3, pp. T115-T140, 2013.

[27] L. Chen, B. Wang, S. Wang et al., "OPMSP: A computational method integrating protein interaction and sequence information for the identification of novel putative oncogenes," Protein and Peptide Letters, vol. 23, no. 12, pp. 1081-1094, 2016.

[28] Y.-H. Huang, C.-H. Liao, R.-N. Chen, C.-J. Liao, and K.-H. Lin, "Human testicular orphan receptor 4 enhances thyroid hormone receptor signaling," Journal of Cellular Physiology, vol. 222, no. 2, pp. 347-356, 2010.

[29] C.-R. Shyr, Y.-C. Hu, E. Kim, and C. Chang, "Modulation of estrogen receptor-mediated transactivation by orphan receptor TR4 in MCF-7 cells," The Journal of Biological Chemistry, vol. 277, no. 17, pp. 14622-14628, 2002.

[30] J. Zhu, D.-R. Yang, Y. Sun et al., “TR4 nuclear receptor alters the prostate cancer $\mathrm{CD} 133^{+}$stem/progenitor cell invasion via modulating the EZH2-related metastasis gene expression," Molecular Cancer Therapeutics, vol. 14, no. 6, pp. 1445-1453, 2015.

[31] L. Du, M. Bergsneider, L. Mirsadraei et al., "Evidence for orphan nuclear receptor TR4 in the etiology of Cushing disease," Proceedings of the National Acadamy of Sciences of the United States of America, vol. 110, no. 21, pp. 8555-8560, 2013.

[32] B. Mao, Z. Zhang, and G. Wang, "BTG2: A rising star of tumor suppressors (Review)," International Journal of Oncology, vol. 46, no. 2, pp. 459-464, 2015.

[33] Y.-J. Zhang, L. Wei, M. Liu et al., "BTG2 inhibits the proliferation, invasion, and apoptosis of MDA-MB-231 triple-negative breast cancer cells," Tumor Biology, vol. 34, no. 3, pp. 1605-1613, 2013.

[34] J. Sima, B. Zhang, X. Y. Sima, and Y. X. Mao, “Overexpression of BTG2 suppresses growth, migration, and invasion of human renal carcinoma cells in vitro," Neoplasma, vol. 63, no. 3, pp. 385-393, 2016.

[35] H. Chen, H. Pan, Y. Qian, W. Zhou, and X. Liu, "MiR-25-3p promotes the proliferation of triple negative breast cancer by targeting BTG2," Molecular Cancer, vol. 17, no. 1, 2018.

[36] S. W. Davis, F. Castinetti, L. R. Carvalho et al., "Molecular mechanisms of pituitary organogenesis: In search of novel regulatory genes," Molecular and Cellular Endocrinology, vol. 323, no. 1, pp. 4-19, 2010.

[37] J. Ando, M. Saito, J.-I. Imai et al., "TBX19 is overexpressed in colorectal cancer and associated with lymph node metastasis," 
Fukushima Journal of Medical Science, vol. 63, no. 3, pp. 141-151, 2017.

[38] Y.-F. Chen, J.-Y. Wang, C.-H. Wu, F.-M. Chen, T.-L. Cheng, and S.-R. Lin, "Detection of circulating cancer cells with K-ras oncogene using membrane array," Cancer Letters, vol. 229, no. 1, pp. 115-122, 2005.

[39] A.-M. Pulichino, S. Vallette-Kasic, J. P.-Y. Tsai, C. Couture, Y. Gauthier, and J. Drouin, "Tpit determines alternate fates during pituitary cell differentiation," Genes \& Development, vol. 17, no. 6, pp. 738-747, 2003.

[40] J. Drouin, "Epigenetic mechanisms of pituitary cell fate specification," in Stem Cells in Neuroendocrinology, pp. 113-121, Springer, 2016.

[41] E. Sjöstedt, J. Bollerslev, J. Mulder, C. Lindskog, F. Pontén, and O. Casar-Borota, "A specific antibody to detect transcription factor T-Pit: a reliable marker of corticotroph cell differentiation and a tool to improve the classification of pituitary neuroendocrine tumours," Acta Neuropathologica, vol. 134, no. 4, pp. 675-677, 2017.

[42] T. A. Chohan, H. Qian, Y. Pan, and J.-Z. Chen, "Cyclindependent kinase-2 as a target for cancer therapy: Progress in the development of CDK2 inhibitors as anti-cancer agents," Current Medicinal Chemistry, vol. 22, no. 2, pp. 237-263, 2015.

[43] C. Caillava, R. Vandenbosch, B. Jablonska et al., "Cdk2 loss accelerates precursor differentiation and remyelination in the adult central nervous system," The Journal of Cell Biology, vol. 193, no. 2, pp. 397-407, 2011.

[44] R. Vandenbosch, L. Borgs, P. Beukelaers et al., "CDK2 is dispensable for adult hippocampal neurogenesis," Cell Cycle, vol. 6, no. 24, pp. 3065-3069, 2007.

[45] S. R. McCurdy, M. Pacal, M. Ahmad, and R. Bremner, "A CDK2 activity signature predicts outcome in CDK2-low cancers," Oncogene, vol. 36, no. 18, pp. 2491-2502, 2017. 


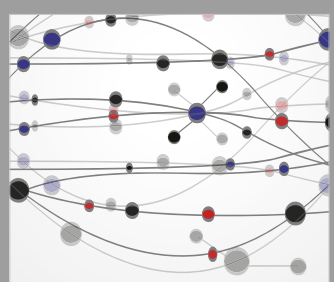

The Scientific World Journal
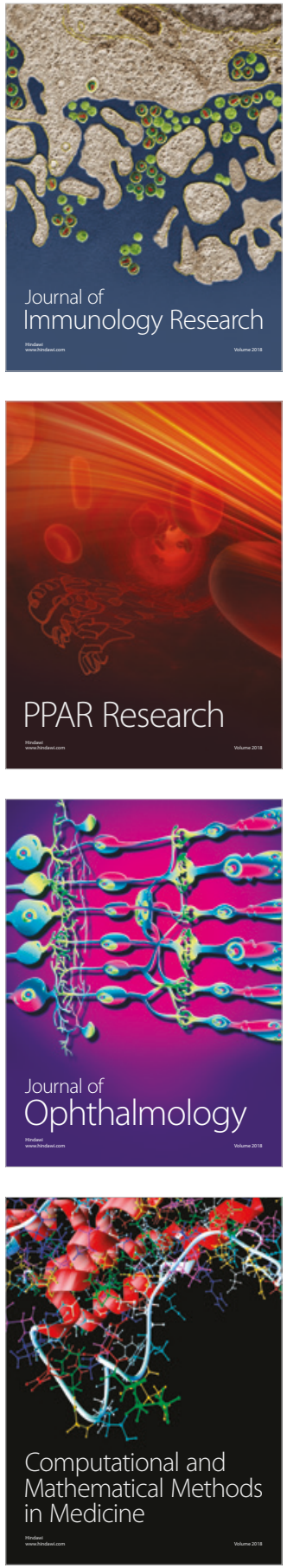

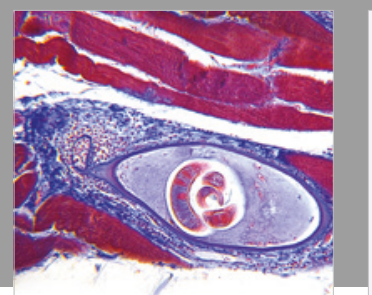

Gastroenterology Research and Practice

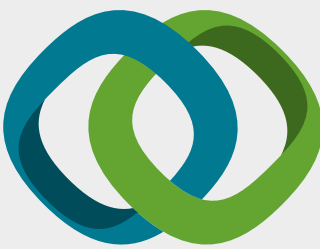

\section{Hindawi}

Submit your manuscripts at

www.hindawi.com
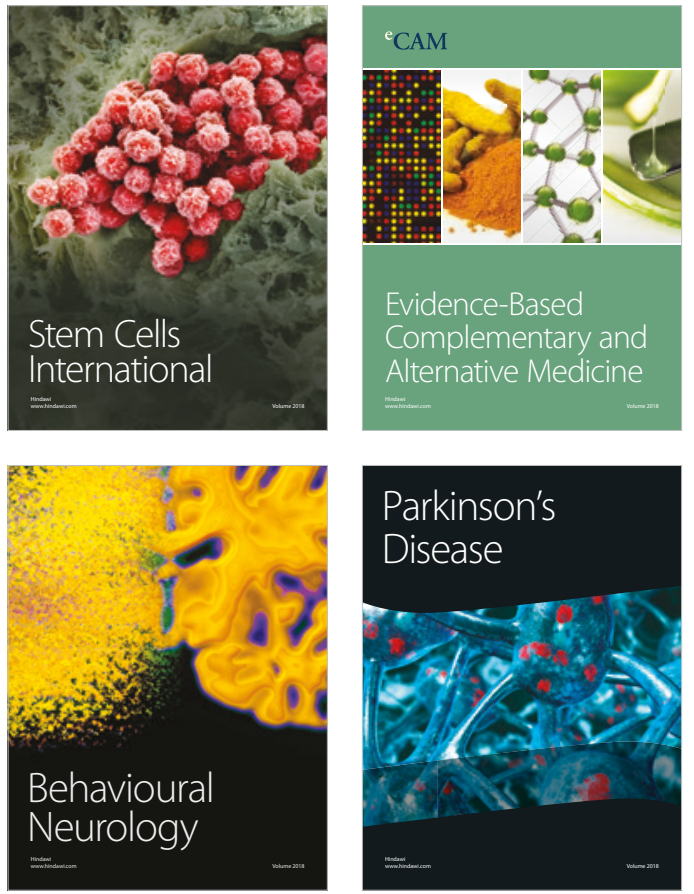

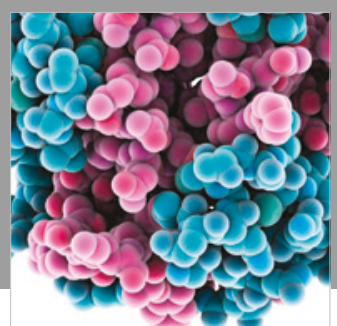

ournal of

Diabetes Research

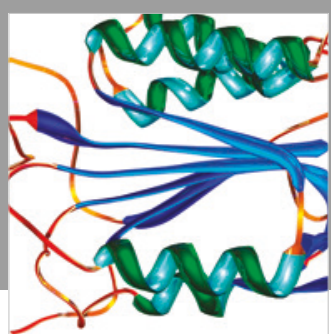

Disease Markers
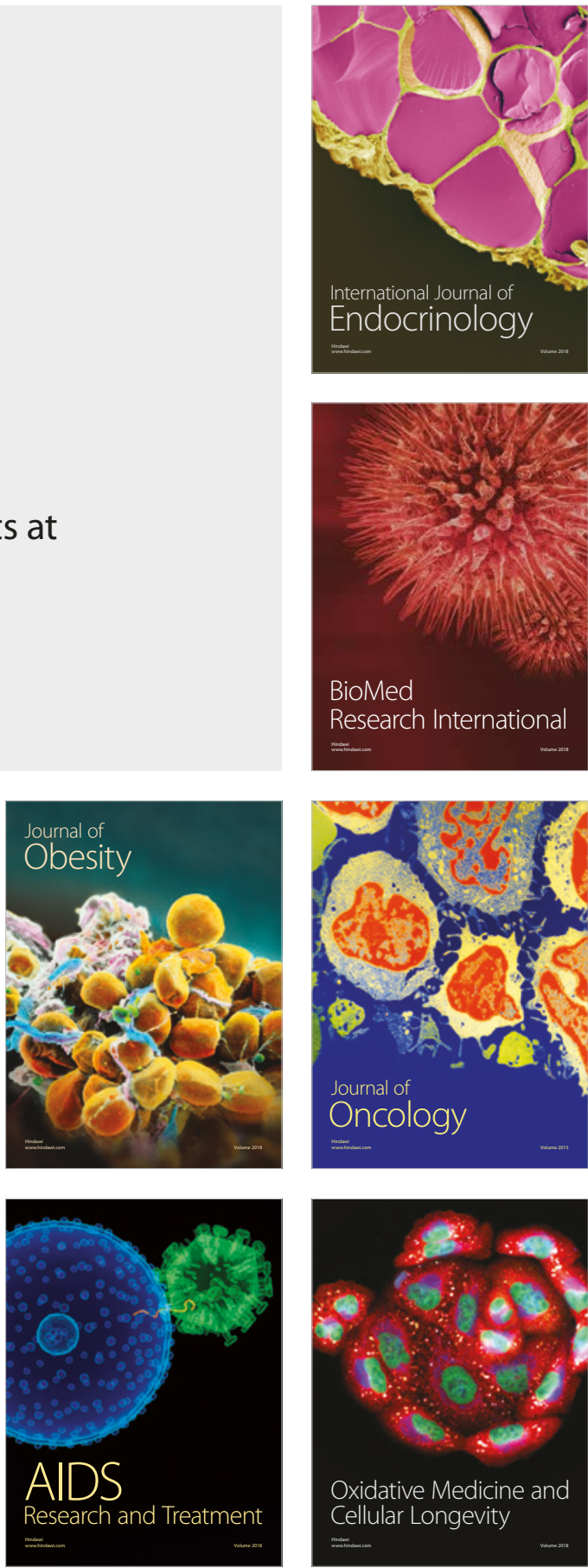